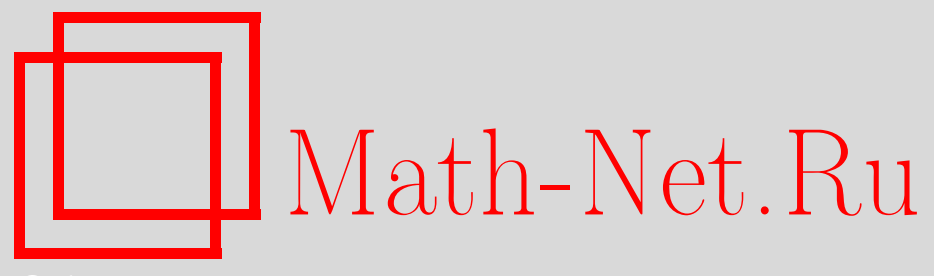

Г. В. Завизион, И. Г. Ключник, Система дифференциальных уравнений с аналитической нелинейностью, $M a-$ тем. заметки, 2012, том 91, выпуск 4, 621-623

DOI: https://doi.org/10.4213/mzm9064

Использование Общероссийского математического портала Math-Net.Ru подразумевает, что вы прочитали и согласны с пользовательским соглашением http://www . mathnet.ru/rus/agreement

Параметры загрузки:

IP : 35.174 .16 .151

26 апреля 2023 г., 16:50:32

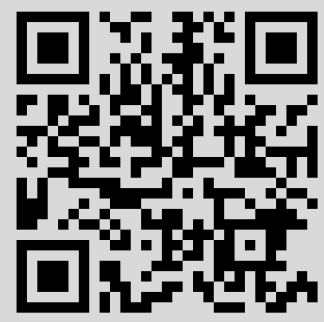




\section{Система дифференциальных уравнений с аналитической нелинейностью}

\section{Г. В. Завизион, И. Г. Ключник}

В [1], [2] проведена локальная линеаризация и выведены условия сходимости линеаризуючего преобразования сингулярно возмущенного дифференциального уравнения первого порядка с аналитичностью по всем переменным и параметром. В [3] методом регуляризации строились асимптотические решения системы сингулярно возмущенных дифференциальных уравнений с аналитической нелинейностью. В данной статье предлагаются методы асимптотического интегрирования задачи Коши для сингулярно возмущенной системы дифференциальных уравнений с аналитической нелинейностью в виде сходящегося ряда экспонент.

Рассмотрим систему дифференциальных уравнений вида

$$
\varepsilon \frac{d x}{d t}=A(t, \varepsilon) x+f(t, \varepsilon, x),
$$

с начальным условием

$$
x(0, \varepsilon)=x_{0},
$$

где $\varepsilon$ - малый параметр, $f(t, \varepsilon, x), x(t, \varepsilon), x_{0}-n$-мерные векторы, $A(t, \varepsilon)-(n \times n)$-матрица. Предполагаем выполнение следующих условий:

1) вектор $f(t, \varepsilon, x)$ имеет разложения в равномерно сходящийся ряд

$$
f(t, \varepsilon, x)=\sum_{|r|=2}^{\infty} a_{r}(t, \varepsilon) x^{r},
$$

где $a_{r}(t, \varepsilon)$ - $n$-мерные векторы, $x^{r}=x_{1}^{r_{1}} x_{2}^{r_{2}} \cdots x_{n}^{r_{n}}, x_{i}$ - компоненты вектора $x(t, \varepsilon)$, $\left|x_{i}\right|<R, r_{i}, i=1, \ldots, n,-$ целые неотрицательные числа, $|r|=\sum_{i=1}^{n} r_{i} ;$

2) $A(t, \varepsilon), a_{r}(t, \varepsilon)$ имеют разложения по степеням малого параметра

$$
A(t, \varepsilon)=\sum_{s=0}^{\infty} \varepsilon^{s} A_{s}(t), \quad a_{r}(t, \varepsilon)=\sum_{s=0}^{\infty} \varepsilon^{s} a_{r s}(t) ;
$$

3) $A_{s}(t), a_{r s}(t), s=0,1 \ldots$, бесконечно дифференцируемы на отрезке $[0 ; L]$;

4) корни $\lambda_{i 0}(t), i=1, \ldots, n$, характеристического уравнения простые.

Справедливы следующие теоремы.

Теорема 1. Если выполняются условия 1)-4) и

$$
\sum_{j=1}^{n} r_{j} \lambda_{j 0}(t) \neq \lambda_{i 0}(t), \quad i=1, \ldots, n, \quad|r| \geqslant 2,
$$

то для любого вектора $x_{0}=\left(x_{01}, x_{02}, \ldots, x_{0 n}\right)$ такого, что $\left|x_{0 i}\right|<\delta, 0<\delta<R, i=1, \ldots, n$, формальное решение задачи Коши (1), (2) имеет вид

$$
x(t, \varepsilon)=\sum_{i=1}^{n} u_{1 i}(t, \varepsilon) \exp \left(\frac{1}{\varepsilon} \int_{0}^{t} \lambda_{i}(t, \varepsilon) d t\right) c_{i}(\varepsilon)+\sum_{|r|=2}^{\infty} u_{r}(t, \varepsilon) \exp \left(\frac{1}{\varepsilon} \int_{0}^{t}(r, \lambda(t, \varepsilon)) d t\right) c^{r},
$$

где

$$
(r, \lambda(t, \varepsilon))=\sum_{j=1}^{n} r_{j} \lambda_{j}(t, \varepsilon), \quad c=\left(c_{1}(\varepsilon), c_{2}(\varepsilon), \ldots, c_{n}(\varepsilon)\right), \quad c^{r}=c_{1}^{r_{1}}(\varepsilon) \cdots c_{n}^{r_{n}}(\varepsilon),
$$


$u_{r}(t, \varepsilon), u_{1 i}(t, \varepsilon)$ - $n$-мерные векторы, $\lambda_{i}(t, \varepsilon)$ - скалярные функиии, которые имеют разложения

$$
u_{1 i}(t, \varepsilon)=\sum_{s=0}^{\infty} \varepsilon^{s} u_{1 i s}(t), \quad \lambda_{i}(t, \varepsilon)=\sum_{s=0}^{\infty} \varepsilon^{s} \lambda_{i s}(t), \quad u_{r}(t, \varepsilon)=\sum_{s=0}^{\infty} \varepsilon^{s} u_{r s}(t) .
$$

ДокАзАтЕЛЬСтво. Подставляя (5) в (1) и сравнивая выражения, которые имеют одинаковые аргументы, получим уравнения

$$
\begin{gathered}
\varepsilon u_{1 i}^{\prime}(t, \varepsilon)+\lambda_{i}(t, \varepsilon) u_{1 i}(t, \varepsilon)=A(t, \varepsilon) u_{1 i}(t, \varepsilon), \quad i=1, \ldots, n, \\
\varepsilon u_{r}^{\prime}(t, \varepsilon)+(r, \lambda(t, \varepsilon)) u_{r}(t, \varepsilon)=A(t, \varepsilon) u_{r}(t, \varepsilon)+f_{r}(t, \varepsilon),
\end{gathered}
$$

где вектор $f_{r}(t, \varepsilon)$ определяется по $a_{l}(t, \varepsilon), 2 \leqslant|l| \leqslant|r|$, в произведении компонент векторов $u_{j_{1}},(t, \varepsilon), u_{j_{2}}(t, \varepsilon), \ldots, u_{j_{|l|}}(t, \varepsilon), j_{i}=0, \ldots,|l|, i=1, \ldots,|l|$.

Учитывая (5), решение уравнения (6) находится методом из [4]. Разложив $f_{r}(t, \varepsilon)=$ $\sum_{s=0}^{\infty} \varepsilon^{s} f_{r s}(t)$ и воспользовавшись $(3),(6),(7)$, получим

$$
u_{r s}(t)=\left(A_{0}(t)-\left(\sum_{j=1}^{n} r_{j} \lambda_{j 0}(t)\right) E\right)^{-1} b_{r s}(t),
$$

где

$$
\begin{gathered}
f_{r s}(t)=\left.\frac{\partial^{s} f_{r}(t, \varepsilon)}{s ! \partial \varepsilon^{s}}\right|_{\varepsilon=0} \\
b_{r s}(t)=-u_{r, s-1}^{\prime}(t)+\sum_{k=1}^{s} \sum_{j=1}^{n} r_{j} \lambda_{j k}(t) u_{r, s-k}(t)-\sum_{k=1}^{s} A_{k}(t) u_{r, s-k}(t)-f_{r s}(t),
\end{gathered}
$$

при $|r| \geqslant 2, s=0,1, \ldots$. Далее, $c_{i}(\varepsilon)$ будем искать в виде $c_{i}(\varepsilon)=\sum_{s=0}^{\infty} \varepsilon^{s} c_{i s}, i=1, \ldots, n$, и раскладывая $c^{r}=\sum_{j=0}^{\infty} \varepsilon^{j} \alpha_{r j}\left(c_{i 0}, c_{i 1}, \ldots, c_{i j}\right)$, а потом подставляя в $(2)$, получим

$$
\begin{gathered}
\sum_{i=1}^{n} u_{1 i 0}(0) c_{i 0}+\sum_{|r|=2}^{\infty} c_{0}^{r} u_{r 0}(0)=x_{0}, \\
\sum_{i=1}^{n} \sum_{j=0}^{s} u_{1 i j}(0) c_{i, s-j}+\sum_{|r|=2}^{\infty} \sum_{j=0}^{s} u_{r j}(0) \alpha_{r, s-j}\left(c_{i 0}, c_{i 1}, \ldots, c_{i, s-j}\right)=0, \quad s=1,2, \ldots,
\end{gathered}
$$

где

$$
\alpha_{r 0}=c_{0}^{r}=c_{10}^{r_{1}} \ldots c_{n 0}^{r_{n}}, \quad \alpha_{r j}\left(c_{i 0}, c_{i 1}, \ldots, c_{i j}\right)=\left.\frac{1}{j !} \frac{d^{j} c^{r}}{d \varepsilon^{j}}\right|_{\varepsilon=0} .
$$

В силу того, что $u_{1 i 0}(t), i=1, \ldots, n,-$ собственные векторы матрицы $A_{0}(t)$, по теореме о неявной функции существует единственное решение уравнения (8) относительно $c_{i s}, s=$ $0,1, \ldots$, в некоторой окрестности точки $\left\{x_{0}|| x_{0 i} \mid<\delta\right\}$. Теорема доказана.

Теорема 2. Если выполняются условия теоремы 1 и неравенства

$$
\operatorname{Re} \lambda_{i 0}(t)<\alpha<0, \quad i=1, \ldots, n, \quad t \in[0 ; L],
$$

то ряд (4) абсолютно равномерно сходится на некотором подмножестве $[0 ; L]$.

Теорема 3. Если выполняются условия 1)-4) и условие: существуют иелые неотрицательные числа $r_{j}^{(0)}, j=1, \ldots, n,\left|r^{(0)}\right| \geqslant 2$ такие, что выполняются соотношения

$$
\sum_{j=1}^{n} r_{j}^{(0)} \lambda_{j 0}(t) \equiv \lambda_{k_{0}}(t), \quad 1 \leqslant k_{0} \leqslant n
$$


а при других значениях $r_{j}^{(1)}$

$$
\sum_{j=1}^{n} r_{j}^{(1)} \lambda_{j 0}(t) \neq \lambda_{k}(t), \quad k=1, \ldots, n, \quad k \neq k_{0}, \quad t \in[0 ; L],
$$

то задача Коши (1),(2) имеет формальное решение вида (4), в котором векторы $u_{1 i}(t, \varepsilon)$, $i=1, \ldots, n$, имеют разложения

$$
u_{1 i}(t, \varepsilon)=\sum_{s=0}^{\infty} \varepsilon^{s+1} u_{1 i s}(t),
$$

a $\lambda_{i}(t, \varepsilon), u_{r}(t, \varepsilon),|r| \geqslant 2$, имеют разложения (5).

Теорема 4. Если выполняются условия 1)-4) и соотношения (3), то формалъное решение задачи Коши (1),(2) имеет вид (5), в котором скалярные функиии $\lambda_{i}(t, \varepsilon)$ и векторы $u_{1 i}(t, \varepsilon), i=1, \ldots, n$, имеют разложения (5), а $c_{i}(\varepsilon)$ удовлетворяют соотношению

$$
\sum_{i=1}^{n} u_{1 i}(0, \varepsilon) c_{i}(\varepsilon)=x_{0}
$$

Таким образом, построены асимптотические решения задачи Коши сингулярно возмущенной системы дифференциальных уравнений с аналитической нелинейностью.

\section{СПИСОК ЦИТИРОВАННОЙ ЛИТЕРАТУРЫ}

[1] Y. Sibuya, J. Math. Mech., 9 (1960), 369-397. [2] В. В. Басов, Вестн. С.-Петербурск. ун-та. Сер. 1. Матем., мех., астрон., 1993, № 2, 62-66. [3] С. А. Ломов, В. Ф. Сафонов, Матем. заметки, 25:6 (1979), 871-889. [4] А. М. Самойленко, М. І. Шкіль, В. П. Яковець, Лінійні системи диференціальних рівнянь з виродженнями, Вища школа, Київ, 2000.

Г. В. Завизион

Кировоградский государственный педагогический университет им. В. Винниченко, Украина

E-mail: ZavizionG@rambler.ru

\section{И. Г. Ключник}

Кировоградский государственный педагогический университет им. В. Винниченко, Украина

E-mail: klyuchnyk.i@mail.ru
Поступило

20.02.2011

Исправленный вариант

10.06.2011 